\title{
A!
}

This is an electronic reprint of the original article.

This reprint may differ from the original in pagination and typographic detail.

Sisto, Renata; Moleti, Arturo; Altoe, Alessandro

Decoupling the level dependence of the basilar membrane gain and phase in nonlinear cochlea models

Published in:

Journal of the Acoustical Society of America

DOI:

$10.1121 / 1.4928291$

Published: 01/01/2015

Document Version

Publisher's PDF, also known as Version of record

Please cite the original version:

Sisto, R., Moleti, A., \& Altoe, A. (2015). Decoupling the level dependence of the basilar membrane gain and phase in nonlinear cochlea models. Journal of the Acoustical Society of America, 138(2), pp. EL155-EL160. https://doi.org/10.1121/1.4928291

This material is protected by copyright and other intellectual property rights, and duplication or sale of all or part of any of the repository collections is not permitted, except that material may be duplicated by you for your research use or educational purposes in electronic or print form. You must obtain permission for any other use. Electronic or print copies may not be offered, whether for sale or otherwise to anyone who is not an authorised user. 


\section{Decoupling the level dependence of the basilar membrane gain and phase in nonlinear cochlea models}

Renata Sisto, Arturo Moleti, and Alessandro Altoè

Citation: The Journal of the Acoustical Society of America 138, EL155 (2015); doi: 10.1121/1.4928291

View online: https://doi.org/10.1121/1.4928291

View Table of Contents: http://asa.scitation.org/toc/jas/138/2

Published by the Acoustical Society of America

\section{Articles you may be interested in}

Compensating for ear-canal acoustics when measuring otoacoustic emissions

The Journal of the Acoustical Society of America 141, 515 (2017); 10.1121/1.4973618

Characterizing spontaneous otoacoustic emissions across the human lifespan

The Journal of the Acoustical Society of America 141, 1874 (2017); 10.1121/1.4977192

A review of otoacoustic emissions

The Journal of the Acoustical Society of America 89, 2027 (1991); 10.1121/1.400897

Estimating cochlear tuning dependence on stimulus level and frequency from the delay of otoacoustic emissions The Journal of the Acoustical Society of America 140, 945 (2016); 10.1121/1.4960588

A cochlear frequency-position function for several species-29 years later

The Journal of the Acoustical Society of America 87, 2592 (1990); 10.1121/1.399052

Distortion product otoacoustic emission generation mechanisms and their dependence on stimulus level and primary frequency ratio

The Journal of the Acoustical Society of America 139, 658 (2016); 10.1121/1.4941248 


\title{
Decoupling the level dependence of the basilar membrane gain and phase in nonlinear cochlea models
}

\author{
Renata Sisto \\ Department of Occupational and Environmental Medicine, Epidemiology and Hygiene, \\ INAIL, National Research Centre for Safety and Prevention at Workplace, \\ Monteporzio Catone, Rome, Italy \\ r.sisto@inail.it \\ Arturo Moletia) \\ Department of Physics, University of Rome "Tor Vergata," Rome, Italy \\ moleti@roma2.infn.it \\ Alessandro Altoè \\ Department of Signal Processing and Acoustics, School of Electrical Engineering, \\ Aalto University, P.O. Box 13000, FI-00076 Aalto, Finland \\ alessandro.altoe@aalto.fi
}

\begin{abstract}
In animal experiments, the strong dependence on stimulus level of the basilar membrane gain and tuning is not matched by a corresponding change in the phase slope in the resonant region. Linear models, in which the gain dependence on the stimulus level has to be schematized by explicitly changing the tuning parameters of the resonant model, do not easily match this feature of the experimental data. Nonlinear models predict a phase slope that is relatively decoupled from tuning. In addition, delayed-stiffness and feed-forward models also show a significant intrinsic decoupling between gain and tuning, which helps in matching the experimental data.

(C) 2015 Acoustical Society of America

[BLM]

Date Received: May 18, 2015 Date Accepted: July 29, 2015
\end{abstract}

\section{Introduction}

Several basilar membrane (BM) vibration experiments on chinchillas and guinea pigs (e.g., reviewed in Robles and Ruggero, 2001) have consistently shown that, at least in the base of the cochlea, although the cochlear gain level and bandwidth strongly depend on the stimulus level in a wide compressive range, the phase-gradient delay in the resonant region only weakly does; in other words, the phase behaviour of the BM resonant response is not very sensitive to the effective gain and tuning factor of the resonance. In the cochlear base, a gain decrease of order $30-40 \mathrm{~dB}$ is accompanied by a relative change of the phase slope of only $25 \%-40 \%$ (roughly estimated from Figs. 5 and 8 of Robles and Ruggero, 2001). This observation may be unexpected because in simple linear single-tuned resonant systems the amplitude and phase of the response are linked by their mutual dependence on the resonance quality factor.

In a previous study (Sisto and Moleti, 2015) a simple passive 1-day transmission line cochlear model, two linear delayed-stiffness active models, and a fully nonlinear model were considered, to investigate this issue. In fully nonlinear cochlear models, it is possible to study the BM response as a function of the stimulus level, whereas in linear models one has to explicitly change the gain of the response, either acting directly on the passive quality factor, or suitably displacing the poles of the resonance in the complex plane to get the same result. It was demonstrated that in the Zweig (Zweig, 1991) and Talmadge (Talmadge et al., 1998) formulations, active delayedstiffness models predict a phase-gradient delay that rapidly increases with increasing quality factor, whereas the passive models predicted dependence as the square root of the quality factor. In a specific fully nonlinear anti-damping (AD) cochlear model (Moleti et al., 2009), directly solved in time domain using the state-space formalism (Elliott et al., 2007), the phase-gradient delay growth was much slower than the tuning growth, increasing approximately as $Q^{1 / 4}$ (Sisto and Moleti, 2015), the estimate of the

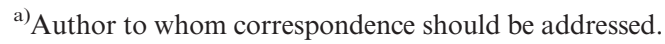


exponent depending on the region in which the derivative is measured, and on the definition of $Q$ (e.g., $Q 3$ is defined relative to the width of the resonance $3 \mathrm{~dB}$ below the peak level). This partial decoupling between phase slope and bandwidth (and gain) qualitatively agrees with animal experiments, suggesting that the experimental BM phase behaviour relies on the intrinsic nonlinear nature of the cochlear dynamics.

In this paper, the relation between stimulus level, gain, and phase-gradient delay has been further investigated using other nonlinear models, to understand to what extent the slower dependence of the phase on gain and tuning was a feature of that specific nonlinear model. To compare more directly the results of the simulations to the experimental data, a slightly different approach was used: the stimulus level range (in the nonlinear models) and the $Q$ range (in their linear versions) were chosen to get a gain range of order $30 \mathrm{~dB}$, and the change of the phase gradient was measured over this parameter range. Nonlinear versions of the Zweig model and of a feedforward (FF) model [see Sisto et al. (2010), following Lim and Steele (2002)], were numerically solved in a wide stimulus range, and compared with the corresponding linear models, in which a similar gain change was obtained by explicitly changing the tuning parameters of the resonance.

\section{Methods and models}

\subsection{State-space nonlinear models}

A fully nonlinear numerical solution scheme was used, which is described in detail in Moleti et al. (2009). The cochlea is schematized by a box-model, with a tonotopic BM separating two volumes filled by a homogeneous incompressible fluid. The set of equations governing the propagation of the differential pressure $p$ and of the transverse BM displacement along the longitudinal direction $x$ is given by

$$
\left\{\begin{array}{l}
\frac{\partial^{2} p(x, \omega)}{\partial x^{2}}=k_{0}^{2} \sigma_{b m} \ddot{\xi}(x, t) \\
\frac{p(x, t)+q(x, t)}{\sigma_{b m}}=\ddot{\xi}(x, t)+\gamma_{b m}(x) \dot{\xi}(x, t)+\omega_{b m}^{2}(x) \xi(x, t),
\end{array}\right.
$$

where $\xi(x, t)$ is the BM transverse displacement, $k_{0}$ is a characteristic inverse length, dependent on the geometry of the cochlea, $\sigma_{b m}$ is the BM surface density, $\gamma_{b m}$ and $\omega_{b m}$ are the damping and stiffness functions, dependent on $x$ according to the Greenwood map (Greenwood, 1990), and $q(x, t)$ the additional pressure applied on the BM by the outer hair cell (OHC) active feedback.

Moleti et al. (2009) considered a diagonal model in which the OHC's force acts locally as an AD term

$$
q(x)=\alpha(x, \dot{\xi}, t) \dot{\xi}(x, t),
$$

and a FF (see Lim and Steele, 2002) model, in which the OHCs' force is proportional to the total pressure at a slightly basally displaced place

$$
q(x)=\alpha(x, \dot{\xi}, t)(p(x-\Delta x, t)+q(x-\Delta x, t)) .
$$

In both cases, $\alpha$ is a saturating nonlinear function, which may either be nonlocal (as in Moleti et al., 2009), or local

$$
\alpha(x, \dot{\xi}, t)=\gamma\left(1-\tanh \left(\frac{\dot{\xi}^{2}(x, t)}{\dot{\xi}_{\text {sat }}^{2}}\right)\right),
$$

yielding nonlinear gain. $\dot{\xi}_{\text {sat }}$ is the BM velocity at which nonlinear saturation effects start. For $\dot{\xi} \rightarrow 0, \alpha \rightarrow 1$, and the maximum gain of the OHC mechanism is achieved. In the opposite limit, $\alpha$ goes to zero, yielding the constant damping regime of a passive system. In the intermediate range, nonlinear compression occurs.

In the discretized model, the dynamic variables representing the $N$ micromechanical elements are embedded in a single state vector $U$ of dimension $2 N$. The above set of equations can be translated in a matrix form

$$
M \dot{U}(t)=A_{E} U(t)+B_{E} S(t),
$$

where, in the linear case, $A_{E}$ is a block-diagonal system matrix, containing the dynamics of the single tonotopic oscillator 


$$
A_{E, i}=\left[\begin{array}{cc}
-\gamma_{b m}(x) & -\omega_{b m}^{2}(x) \\
1 & 0
\end{array}\right]
$$

where $S(t)$ represents a stimulus term applied to the stapes, and $M$ represents the mass matrix of the system. In the linear case, and in the nonlinear AD case, $M$ is linear, and is represented by the operator

$$
M=M_{\text {diag }}=I-B_{E} F^{-1} C_{E},
$$

where $F$ is the discretized Laplace operator, and the matrices $B_{E}$ and $C_{E}$ are projection operators, defined in Elliott et al. (2007), picking up, respectively, the displacement and velocity components of the state space vector $U$. In the nonlinear $\mathrm{AD}$ case, the nonlinear term is added to the right side of Eq. (6) as

$$
B_{E} D D_{E} U(t)
$$

where $D$ is a diagonal matrix such that $D(i, i)=\alpha\left(x_{i}, \xi, t\right)$. The operator $D_{E}$ is equal to $C_{E}$ multiplied by the function $\gamma_{b m}(x)$. In the FF case, we put the nonlinear term in the mass matrix

$$
M_{F F}=I-B_{E} G(U) F^{-1} C_{E} .
$$

$G(U)$ is a nonlinear gain matrix: $G(U)=B^{-1}$, whereas the matrix $B$ has ones on the main diagonal and non-null off-diagonal terms

$$
B(i+K, i)=-\alpha\left(x_{i}, \xi, t\right),
$$

where $K(=1)$ is the number of elements corresponding to longitudinal FF shift.

\subsection{Delayed-stiffness nonlinear model}

In the case of the Zweig model, the nonlinearity was schematized by introducing a dependence on the local BM displacement in the term

$$
\Delta(x, \omega)=\omega_{b m}^{2}(x)-\omega^{2}+j \omega \delta+\rho \omega_{0}^{2}(x) e^{-j 2 \pi \mu\left[\omega / \omega_{0}(x)\right]} .
$$

In order to get an effective quality factor dynamically varying, with increasing $\mathrm{BM}$ velocity, between two asymptotically linear regimes with, respectively, $Q 3=Q_{\text {act }}$ and $Q 3=Q_{\text {pass, }}$, we set

$$
Q \equiv Q 3=Q_{\text {pass }}+\left(Q_{\text {act }}-Q_{\text {pass }}\right)\left(1-\tanh \left(\frac{\dot{\xi}^{2}(x, t)}{\dot{\xi}_{\text {sat }}^{2}}\right)\right) .
$$

Starting from an empirical relation (see Sisto and Moleti, 2015) between $Q 3$ and the real part of the Zweig impedance, the parameters of the double pole cochlear admittance $\{\delta, \rho, \mu\}$ are computed at each time step by solving a system of five equations (Shera, 2001), so the poles move in the complex plane to get the desired dependence of the effective quality factor of the model on the instantaneous BM velocity level.

\subsection{Numerical solution}

The FF model has been solved in time domain, using a direct solver in which the mass matrix of the system is on the right side of the equation. A suitable manipulation of the matrices has been used to avoid the inversion of the Laplace operator $F$. In addition, considering its tri-diagonal structure, a convenient factorization of the mass matrix has been introduced. The solution was computed in time domain, using the ode 45 MATLAB solver adapted to stiff problems, on $N=1000$ cochlear elements. A complete state space approach was found indispensable for solving both the AD and FF models described above. Using different solution approaches, the expected dynamical range of the gain was not reproduced for these models.

The complete state space approach was not necessary in the case of the Zweig delayed-stiffness model. In this case, an alternative approach permits to speed up the solution significantly. The equations in Eq. (1) are solved using the decomposition described in Diependaal et al. (1987),

$$
\left\{\begin{array}{l}
A p=g \\
\ddot{\xi}=\frac{p-g}{\sigma_{b m}},
\end{array}\right.
$$


where $g$ is

$$
g=\gamma_{b m}(x) \dot{\xi}+\omega_{b m}^{2}(x) \xi
$$

and $A$ is the matrix representing the pressure coefficient obtained by substituting $\ddot{\xi}$ in the second line of Eq. (1) with a spatially discretized version of the solution of the first line of Eq. (1). Exploiting the fact that $A$ is a tri-diagonal matrix, its inversion is possible by using the Thomas algorithm. The numerical solutions of Eq. (13) are then obtained through a Runge-Kutta standard method, where in each integration sub-step the delayed stiffness terms are computed through spline interpolation to improve both numerical accuracy and computational performance (Altoè et al., 2014).

\section{Results and discussion}

For the simple passive model, and for the AD model, it is sufficient to recall the results of Sisto and Moleti (2015), in which a simple passive 1-day transmission line model showed a phase-gradient delay increasing, as expected, as the square root of $Q$. Therefore, a gain change of order $40 \mathrm{~dB}$ implies, in a simple passive linear model, a change of $Q$ by a factor of 100, and of the phase-gradient delay by a factor of 10 . In the nonlinear AD model, a 35-40 dB change of gain implies a similar change of $Q$, but only a change of the phase-gradient delay by a factor of 3-5 (Sisto and Moleti, 2015). In the nonlinear AD model, an intrinsic relation between gain and bandwidth holds [Eqs. (2) and (4)], but the slope of the phase is partly decoupled from both gain and bandwidth, compared to the corresponding passive model.

In Fig. 1, a significant change of the phase-gradient delay is also shown for the linear active Zweig model and for a linear FF model, in which the tuning factors were explicitly changed to mimic different stimulus level regimes. In both cases, a gain change of $30 \mathrm{~dB}$ corresponds to a phase slope change by a factor of 2 . In such linear models, the coupling between quality factor and phase gradient is still strong, but the relatively smaller phase-gradient delay changes shown in Fig. 1 (compared to the passive case, not shown) are due to the fact that the coupling between $Q$ and gain is different from that of simple passive models, meaning that a large gain change may be obtained with a relatively smaller $Q$ change. Nevertheless, the changes of the phase gradient of Fig. 1 are still larger than those typically found in animal studies of the $\mathrm{BM}$ vibration over a comparable gain range.

In nonlinear models, an additional force is included, associated with the OHC amplifier, with a nonlinear compressive dependence on the BM displacement or velocity, which dynamically changes the gain and tuning of the BM response as a function of the stimulus level. What we observe is that such dynamical changes do not
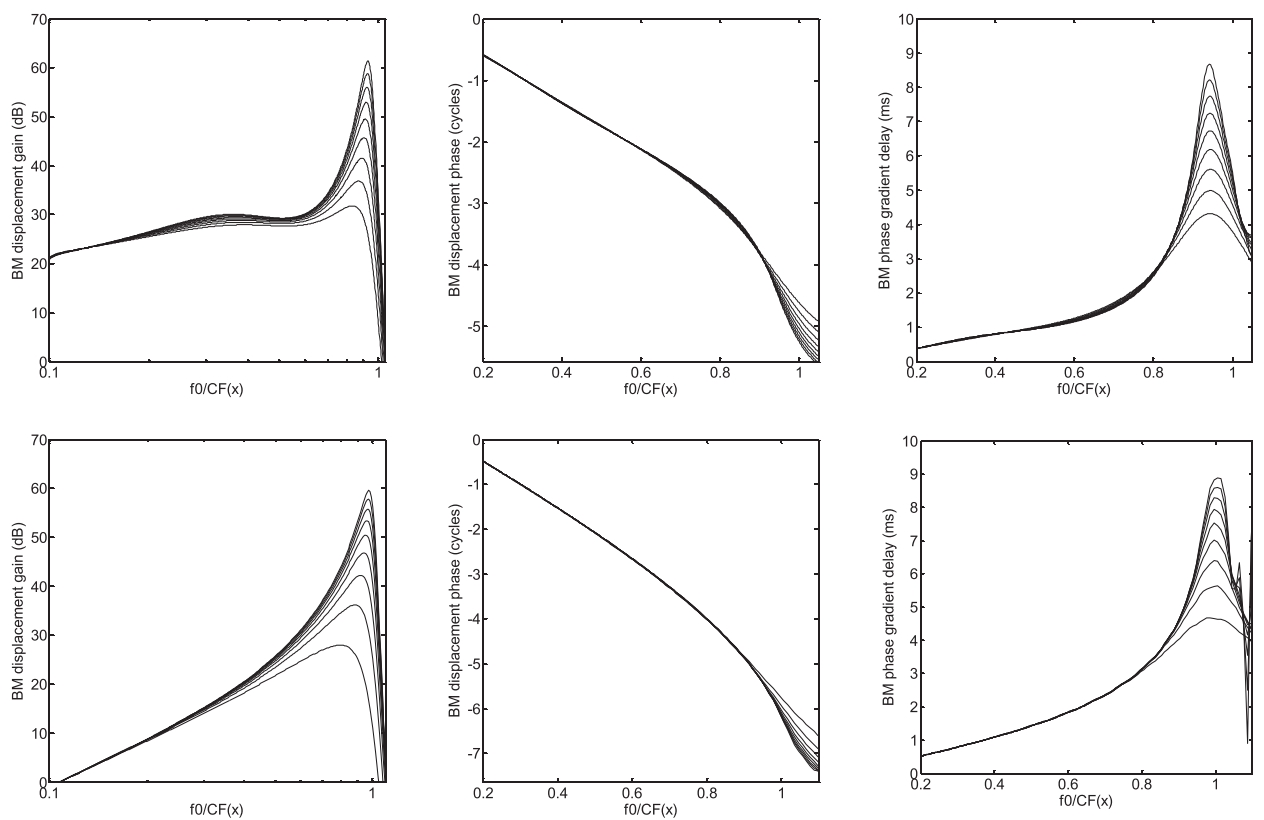

Fig. 1. Gain (left), phase (middle), and phase-gradient delay (right) profiles computed for a Zweig linear model (top) and a FF linear model (bottom), for a sinusoidal stimulus of frequency $f_{0}=2 \mathrm{kHz}$. Here and elsewhere, the dimensionless variable $f_{0} / \mathrm{CF}(x)$ is used for the horizontal scale. A gain change of order $30 \mathrm{~dB}$, obtained by explicitly changing the tuning properties of the resonance, is accompanied by a twofold change in the phasegradient delay, larger than that typically observed in animal BM vibration experiments. 
significantly affect the phase slope in the resonant region, in agreement with experiments, for several different nonlinear models, in a wide stimulus level range. In Fig. 2, a very small phase slope change is shown for the nonlinear FF model, with $Q=8$ and $\gamma=0.5$, level $=20-80 \mathrm{~dB}$, and for the nonlinear Zweig model, level $=10-80 \mathrm{~dB}$. In the nonlinear Zweig case, a gain change of $30 \mathrm{~dB}$ corresponds to a phase slope change of $20 \%$ only, whereas in the linear FF case, the phase-gradient delay changes by less than $10 \%$ for a gain change of $30 \mathrm{~dB}$.

This approximate invariance of the phase profile seems to be related to the property of nonlinear models of changing the gain and tuning properties of the resonance only through the dynamical change of the state variables of the system (BM displacement and/or velocity). In a linear cochlear model, the phase at the resonant place is a scale-invariant quantity, independent of frequency, at the corresponding longitudinal position $x$. At constant place, the phase monotonically decreases as a function of frequency, accumulating most of its lag within the resonance bandwidth. The dual representation of this phenomenology is observed at constant frequency as a function of the longitudinal displacement $x$, with the phase decreasing with decreasing local characteristic frequency $\mathrm{CF}(x)$. By increasing the quality factor, a significant phase lag is increasingly concentrated in a narrow spatial width, corresponding to the frequency bandwidth. As a consequence, the phase-gradient delay becomes a steeper function of $Q$. A similar effect is observed in linear active models, if the tuning and, consequently, the gain, are explicitly changed to mimic different stimulus level regimes. In nonlinear models, in which the additional force is implicitly modified to get the change of gain and bandwidth only through a dynamical nonlinear dependence on the instantaneous BM displacement/velocity, this phase profile invariance is approximately preserved, compared to the corresponding linear case. The steepness of the phase is related to the asymptotic regime of the BM dynamics, valid for small displacements, and it is not significantly altered by the instantaneous compression of the response in the saturated regime. In other words, dynamically changing the effective gain and bandwidth of a resonance in a nonlinear model does not alter the phase function as an explicit change of the $Q$ factor does in a linear model.

One should note that in the FF and Zweig models the phase-gradient delay varies much less than in the AD model, for similar gain changes. This difference between the three nonlinear models depends, as for the corresponding linear models, on the different dependence between gain and $Q$. The same dynamical change of the gain corresponds to different $Q$ ranges for the three models. In the FF model, due to the fact that the additional force is proportional to total force (including itself) at a longitudinally shifted place, a pile-up effect occurs (Moleti et al., 2009; Sisto et al.,
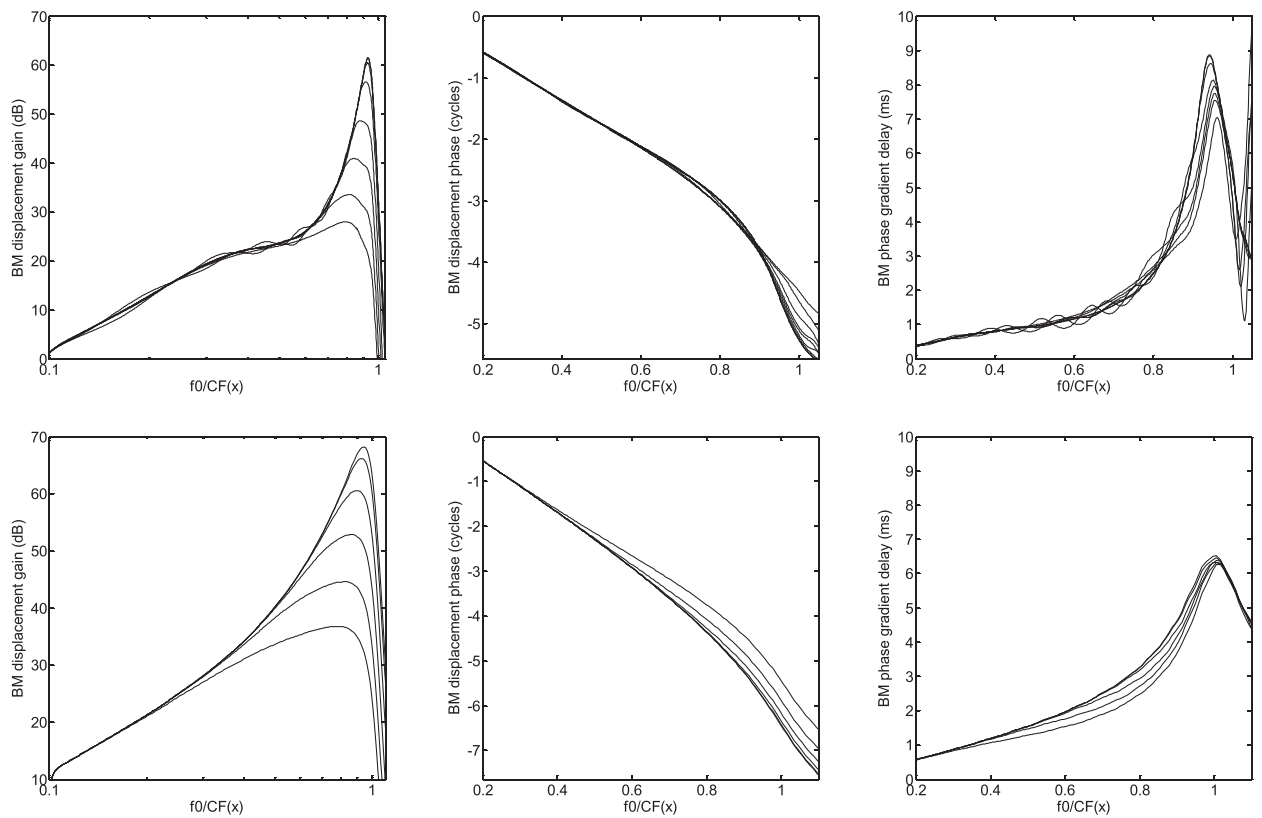

Fig. 2. Gain (left), phase (middle), and phase-gradient delay (right) profiles computed for the nonlinear versions of both models, Zweig (top) and FF (bottom), of Fig. 1, for different stimulus levels, encompassing their compressive nonlinear region. A $30-40 \mathrm{~dB}$ gain change, of the same size as that in Fig. 1, is accompanied by small changes in the phase gradient profiles (respectively, $20 \%$ and less than $10 \%$ ), even smaller than in animal $\mathrm{BM}$ vibration measurements. 
2010), so that a small change in the quality factor can be associated with a large gain change. A similar phenomenon occurs in the Zweig model due to the time-delayed stiffness term. In the AD model, the $\mathrm{OHC}$ gain is directly associated with a local decrease of damping. Therefore gain and tuning are strictly related, almost as in a simple passive linear model. Although the nonlinear model shows a smaller phase-gradient delay than the passive linear model also in this case, the strong coupling between gain and bandwidth explains why the AD model is comparatively less effective in reproducing the experimental approximate insensitivity of the phase-gradient delay to large dynamical changes of the BM gain.

\section{Conclusions}

The observed small variability range of the phase slope of the basal BM response in animal experiments may be explained by nonlinear cochlear models, and it is sensitive to two factors: (1) the different relation between gain and bandwidth implied by different cochlear models, and (2) the dependence of phase slope on bandwidth itself. The second factor is strongly reduced in nonlinear models, compared to the corresponding linear ones. The first factor depends on the mathematical structure of the additional force associated with the $\mathrm{OHC}$ mechanism, and it is strongly reduced in FF and Zweig-like models, with respect to the simple AD model. In the FF and Zweig nonlinear models the phase gradient changed very little (less than $10 \%$ and $20 \%$, respectively, which is even less than in the animal experiments) within the whole nonlinear regime (corresponding, in the simulations, to gain changes of order $30 \mathrm{~dB}$ ), keeping close to that of the asymptotic high gain regime, valid for very low stimulus levels. Although linear models could be suitably designed to match the experimental slow dependence of phase slope on stimulus level, nonlinear dynamics helps to get the same result with less constraints on the model structure.

\section{References and links}

Altoè, A., Pulkki, V., and Verhulst, S. (2014). "Transmission line cochlear models: Improved accuracy and efficiency," J. Acoust. Soc. Am. 136, EL302-EL308.

Diependaal, R. J., Duifhuis, H., Hoogstraten, H. W., and Viergever, M. A. (1987). "Numerical methods for solving one-dimensional cochlear models in the time domain," J. Acoust. Soc. Am. 82, 1655-1666.

Elliott, S. J., Ku, E. M., and Lineton, B. (2007). “A state space model for cochlear mechanics," J. Acoust. Soc. Am. 122, 2759-2771.

Greenwood, D. D. (1990). "A cochlear frequency position function for several species-29 years later," J. Acoust. Soc. Am. 87, 2592-2605.

Lim, K. M., and Steele, C. R. (2002). "A three dimensional nonlinear active cochlear model analyzed by the WKB numeric method," Hear. Res. 170, 190-205.

Moleti, A., Paternoster, N., Bertaccini, D., Sisto, R., and Sanjust, F. (2009). "Otoacoustic emissions in time-domain solutions of nonlinear non-local cochlear models,” J. Acoust. Soc. Am. 126, 2425-2536.

Robles, L., and Ruggero, M. A. (2001). "Mechanics of the mammalian cochlea," Physiol. Rev. 81, $1305-1352$.

Shera, C. A. (2001). "Intensity-invariance of fine time structure in basilar membrane click responses: Implications for cochlear mechanics,” J. Acoust. Soc. Am. 110, 332-348.

Sisto, R., and Moleti, A. (2015). "On the dependence of the BM gain and phase on the stimulus level," in Proceedings of the Mechanics of Hearing 2014 Workshop, Sounio (June 23-29, 2014).

Sisto, R., Moleti, A., Paternoster, N., Botti, T., and Bertaccini, D. (2010). "Different models of the active cochlea, and how to implement them in the state-space formalism," J. Acoust. Soc. Am. 128, 1191-1202.

Talmadge, C. L., Tubis, A., Long, G. R., and Piskorski, P. (1998). "Modeling otoacoustic emission and hearing threshold fine structures," J. Acoust. Soc. Am. 104, 1517-1543.

Zweig, G. (1991). "Finding the impedance of the organ of Corti," J. Acoust. Soc. Am. 89, 1229-1254. 